\title{
Wird unser Land zweisprachig?
}

\section{Vorsichtige Überlegungen zur Geschichte und Zukunft des Deutschen [2008]}

Die deutsche Sprache, unser jüngstes Sorgenkind: Verschwindet sie? Wird sie überformt, auf manchen Gebieten zum Stillstand verurteilt, in ihrem Selbststand das ist Leibniz' (vgl. 1916a: 71) Ausdruck - getroffen? Wird Englisch einmal die Universalsprache, der gegenüber alle anderen Nationalsprachen den Status eines Dialekts annehmen?

Will our future be bilingual?

Are we going to switch between perfect German and perfect English, comparable to Dr. Martin Luther or Erasmus from Rotterdam, who changed between German and Latin as easy as we at home walk between kitchen and hallway? Wird man Sie hier nach einer Generation auf Englisch begrüßen?

Als Cosimo I. von Florenz im Jahr 1541, um sein Ansehen zu steigern und seine öffentliche Stellung zu festigen, die Tore der Universität für die Bürger der Stadt öffnete, verlangte er von den Professoren nicht nur, dass sie diese ihre ,publice‘ gehaltenen Vorlesungen in der Volkssprache zu halten hatten, im toskanischen Volgare, sondern auch, dass sie ihren öffentlichen Vorlesungen eine Stelle aus der Göttlichen Komödie voranstellen und zugrunde legen sollten - ein Wort von Dante.

Wir müssten wohl, wenn wir dem folgen wollen, eines von Goethe wählen. Der anerkannteste Meister unserer Sprache, der mit der größten Reichweite und zugleich vielleicht lebendigste, hat sich nur sehr knapp zu seinem Handwerkzeug geäußert. Es gibt einen Achtzeiler (WA I, 4: 71):

\footnotetext{
Worte sind der Seele Bild -

Nicht ein Bild! sie sind ein Schatten!

Sagen herbe, deuten mild

Was wir haben, was wir hatten. -

Was wir hatten wo ist's hin?

Und was ist's denn was wir haben? -

Nun, wir sprechen! Rasch im Fliehn

Haschen wir des Lebens Gaben.
}

Hier wird Zeile für Zeile etwas gesagt und wieder zurückgenommen. Ist das Wort ein Abbild unseres Innern, oder nur ein Zeichen, definiert es scharf oder deutet es nur freigiebig an? Ist, was es sagt, jetzt da und bereits vorbei? - Die Sprache ist wie ein vorübergehender Hauch und hält dennoch etwas fest. 
Wir hätten auch aus Goethes einzigem Aufsatz zur Sprache zitieren können, der 1816 erschien, und in dem er sich gegen die Sprachreiniger, die Fremdwortverteufler wendet, gegen Sprachnationalismus:

Die Gewalt einer Sprache ist nicht, daß sie das Fremde abweist, sondern daß sie es verschlingt. (Goethe 1907: 207)

Zu der Zeit, als dies Wort fiel, ging es um den Einfluss des Französischen. In den kriegerischen Auseinandersetzungen mit Napoleons Frankreich war ein deutsches Nationalbewusstsein entstanden, das bei einigen Schriftstellern zu dem Programm führte, sämtliche Französismen zu übersetzen. Joachim Heinrich Campes Wörterbuch zur Erklärung und Verdeutschung der unserer Sprache aufgedrungenen fremden Ausdrücke von 1801 und 1813 ging davon aus, dass das Gebrauchsdeutsch seiner Zeit zu einem Fünftel aus Fremdwörtern bestehe und unternimmt oder übernimmt 11.000 Verdeutschungsversuche: es entstand ein ungemein interessantes Wörterbuch. Ich habe öfter darin gelesen. Die meisten Wörter Campes haben sich nicht durchgesetzt. Er übersetzte Mumie mit ,Dörrleiche‘, Kardinal mit ,Purpurpfaff‘, Paradies mit ,Wonnegefilde‘, Souterrain mit ,Erdkammer‘. Den meisten Übersetzungen ging es wie diesen, sie wurden nicht aufgenommen. Aber an die 300 Wörter, die bei ihm in der Übersetzungsspalte stehen, sind uns heute selbstverständlich: Gesetzgebende Versammlung für ,Legislative‘, Minderheit für 'Minorität', verwirklichen für ,realisieren', Tageszeitung für ,Journal', dienstunfähig für ,invalid‘, Stelldichein für ,Rendezvous‘. Sie werden bemerkt haben - die sog. Fremdwörter sind ebenfalls erhalten. Campe, der Hauslehrer der Humboldts, der mit seinen Zöglingen in das Paris der Revolutionsjahre reiste, hat die Sprache bereichert, aber nicht, wie er wollte, zugleich gereinigt von „fremden Wortgut“. Die beiden erhaltenen Wörter haben einen verschiedenen Klang, eine etwas unterschiedene Bedeutung. ,Dienstunfähig“ ist nicht das Gleiche wie ,invalid‘, ein ,Rendezvous' klingt in norddeutschen Ohren viel verruchter als ein ,Stelldichein“. Campes Übersetzungen lassen oft ein feines Ohr erkennen. Etwas komisch wirkt dagegen das knorrige ,Teutsch', das Turnvater Jahn, der wackere Recke, in unsere Sprache einführen wollte: den „Sprung in den Seitstreckhang ristgriffs [...] Unterschwung in den Seitstand rücklings. Oberarmhang und Schwungstemme“ (vgl. Bendix 1922: 88-107).

Wir sprechen aus einer gründlich veränderten geschichtlichen Situation. Das Englische ist auf eher leise und kaum umstrittene Weise zur Weltsprache Nr.1 geworden, zur sog. Lingua franca. Unser Land ist heute in weiten Bereichen der Wissenschaften und der Industrie zweisprachig, auf anderen öffentlichen Gebieten haben die Entlehnungen aus dem Englisch-Amerikanischen so zugenommen, dass der Zustand der deutschen Sprache zu einem privaten und öffentlichen 
Sorgenkind geworden ist. Und wenn man sich vor Augen hält, was über die demographische Entwicklung bekannt ist, dass wir eine jährliche Zuwanderung von 200.000 Personen brauchen, wenn ein derzeitiges Tief aufgefangen werden soll, oder was über ,Migration und Integration“ seit den fünfziger Jahren mitgeteilt wird, dann sind wir längst nicht nur zweisprachig, sondern ein vielsprachiges Land.

Das ist bekannt. Aber vermutlich gilt hier, was im Bereich ,Zuwanderung‘ so auffällig war: es ist zu wenig anerkannt.

Bis in die 1990er Jahre galt die politische Sprachregelung, Deutschland sei ,kein Zuwanderungsland‘. Der verdrängten Tatsache entsprach ein Mangel an Antworten. Das öffentliche Bewusstsein, die Verständigung über die Situation und die Schlussfolgerungen hinken auch heute der eingetretenen Situation hinterher.

Trifft es zu? Wird das Land zweisprachig? Sind wir auf dem Weg zu BSE - Bad Simple English, wie manche behaupten?

Befinden wir uns im Ganzen auf der Straße nach BSG - Bad Simple German? Zur deutschen von Englisch durchsetzten und reduzierten, zur Stummelsprache? $\mathrm{Zu}$ einem verkümmerten Sprachenbabel?

Sind die düsteren Prognosen, die man zeitweise liest oder hört, übertrieben gehen sie von überzeugenden Voraussetzungen aus?

Gestatten Sie zunächst einen Rundblick auf einige Beispiele und Daten:

Wenn Sie in den Ferien auf den Kanarischen Inseln waren, war das Wetter im Vergleich - vielleicht so schön, dass Sie Ihren Flight gecancelt haben und das Ticket verfallen ließen. Auf der Bahnreise fällt es nicht mehr auf, dass wir im Intercity fahren. Auf der Abfahrttafel liest man Departure, im ,Erlebnisbereich“ ,coole drinks‘, auf dem Wegweiser ,Ausgang City‘, wo jetzt das Reisezentrum eingerichtet ist, stand vor einiger Zeit ,Service Point‘.

Wo in der vorigen Generation die Schreibmaschine stand, sind jetzt ,Computer‘ und ,Internet', ,Laptop‘, ,Beamer‘ und ,powerpoint‘ alltäglich, oder auch der ,Internet-Shop'.

Unsere Nachbarn, gestandene Journalisten, spielen einmal in der Woche nicht mehr das, was wir einmal ,Federball‘ nannten, sondern ,Badminton“, sie ,joggen' nicht mehr, sondern fahren per ,Mountainbike‘ auf den Schauinsland, wie blass würde dagegen ,Bergrad' klingen; wir sollten uns ,Nordic Walking‘ überlegen, um ,fit‘ zu bleiben.

Die ,youngsters‘, die ,twens‘, wie sie auch einmal hießen, hatten einmal eine ausgeprägt englische Phase. Da konnte man z.B. auf dem Freiburger Mariensteg folgenden Dialog hören: „Ich bin ausgezogen“, sagt jemand. „Die parents?“ fragt die Schulfreundin. „Nein“, antwortet er. „Meine connections zur family sind total okay." 
Das Amtsblatt der Gemeinde Feldberg lädt am 13. Dezember 2006 auf der linken Spalte zu einer Feldberger-Vereinsweihnacht ein. Hier hat von acht Programmpunkten nur einer einen englischen touch: Der SV Feldberg Cheerleader kommt mit dem Weihnachtsmusical ,Rudolf das Rentier‘.

Auf der rechten Spalte lädt ein ,Qowaz-Feldbergalarm‘ zu einem ,Easy Fun Park‘ ein; von 14 Programmpunkten sind elf englisch formuliert. Die Musikszene spricht Englisch.

Bei Entlehnungen, schreibt der Sprachwissenschaftler Stephen Ullmann (1967: 95), also bei der Zuwanderung von Wörtern aus einer anderen Sprache, ihrer Anleihe, kommt es vor allem auf das Prestige an. Das ist heute so wie zu der Zeit, als in der Küche, Kleidung und Liebe das Französische à la mode war. Auch Mode ist übrigens ein Lehnwort aus dem Französischen. Es bedeutet ,Art und Weise'.

Es ist nicht nur das Prestige, das hier wirkt, es ist das sog. Kulturgefälle, der scheinbare oder tatsächliche Vorsprung der anderen Seite. Die bisherigen Beispiele stammten aus den Bereichen Verkehr und Information, Jugendsprache, Musik und Unterhaltung. Der hier stattfindende Austausch ist begreiflich und findet weltweit statt.

Wo das Prestige, das Kulturgefälle gilt, greift die Werbung zu. Werbung ist Prestigeanleihe, sie bedient sich vor allem des Mittels der Anleihe bei einer Zeichenwelt, die Prestige genießt: bei schönen Frauen und berühmten Männern, wissenschaftlicher Sprache und englischem Vokabular, das am billigsten zu haben ist. Es kostet gar nichts. Werbung ist teuer, und weil Englisch das billigste Mittel der Werbung ist, wird es ein wirksamer Faktor des Sprachwandels.

Man erkennt aber schon an den meisten Beispielen, wie stark das geschichtliche Machtgefälle beteiligt ist, wie das Zusammenwachsen der Welt auf die Nationalsprachen einwirken muss: die Industrieproduktion und Wirtschaft, der Handel wird durch Grenzen kaum aufgehalten. Hier habe ich keine präzisen Zahlen. Aber dass ein Unternehmen wie Daimler in Sindelfingen seinen Schriftwechsel auf englisch führt, ist längst selbstverständlich. Der Deutsche Schraubenverband ist kürzlich zum Englischen übergegangen.

Die Wissenschaft ist per Definition eine internationale Verständigungsgemeinschaft; seit einiger Zeit drückt sich das wieder darin aus, dass sie eine gemeinsame Sprache verwendet. Die große Mehrheit deutscher Wissenschaftler publiziert heute Englisch. Wir haben 1990 die Entwicklung an unserer Freiburger Universität untersucht: eine Gruppe von Germanisten und Biologen versandte einen Fragebogen, unser Kollege Jürgen Schiewe (1991b: 40) fasste das Resultat zusammen: 


\begin{abstract}
Englisch hat sich als Wissenschaftssprache in den Naturwissenschaften (Physik, Chemie, Biologie, Geologie) sowie in der Medizin, der Mathematik und den Wirtschaftswissenschaften schon weitgehend durchgesetzt. Die Fächer der Philosophischen Fakultät, mit Ausnahme der Psychologie, sind noch überwiegend deutschsprachig, aber auch bei ihnen zeigt sich eine deutliche Tendenz zum Englischen. [...] Lediglich in der Rechtswissenschaftlichen Fakultät herrscht Deutsch als Wissenschaftssprache eindeutig vor, was durchgehend mit dem Gegenstand des Faches - dem nationalen Recht - begründet wird.

Wissenschaftler aller Fakultäten sehen sich dem Druck ausgesetzt, Englisch als Wissenschaftssprache benutzen zu müssen. Ungefähr zwei Drittel aller Befragten glauben, daß ihre wissenschaftliche Karriere vom Gebrauch des Englischen abhängig sei.
\end{abstract}

Wenn die Deutsche Akademie für Sprache und Dichtung ihren alljährlichen Sigmund-Freud-Preis für wissenschaftliche Prosa verleihen will, der 1964 zur Förderung einer Textgattung gestiftet wurde, die „bei uns im Vergleich zu anderen europäischen Literaturen nicht gebührend geschätzt und daher auch nicht genügend entwickelt“ (vgl. Handbuch der Kulturpreise. Online-Ausgabe: http://www. kulturpreise.de/web/preise_info.php?cPath=6_103\&preisd_id=838; 03.09.2019) sei, steht sie vor einer schrumpfenden Auswahl möglicher wissenschaftlicher Disziplinen. Die Preisträger Adolf Portmann (1965), Werner Heisenberg (1976) und Carl Friedrich von Weizsäcker (1988) schrieben in der Regel Deutsch, die deutsche Naturwissenschaftlergeneration nach ihnen schreibt in der Regel Englisch.

Das ist bekannt. Doch man kann nicht sagen, dass dieser einschneidende Sprachwandel ganz ins öffentliche Bewusstsein getreten sei, noch weniger, dass es zu einer öffentlichen Debatte, zu wirksamen Überlegungen und Reaktionen in Wissenschaft und Bildung geführt habe. Deutsch war von der Mitte des 19. bis in die 20er Jahre des 20. Jahrhunderts eine in den europäischen Ländern und in der weiteren wissenschaftlichen Welt gelesene Sprache.

Zunächst durch die Vertreibungspolitik der Nazis, dann aber aufgrund der allgemeinen internationalen Entwicklung wurde es, wie auch das Französische oder Russische, eine wissenschaftliche Randsprache. Es trifft, was wir hier beobachtet haben, im Ganzen zu: Englisch ist die Lingua franca der heutigen Wissenschaften. Die Statistik ist eindeutig; die Vorteile leichter internationaler Kommunikation liegen auf der Hand. Wer Deutsch schreibt, hat als Wissenschaftler kaum eine Chance, international wahrgenommen und rezipiert zu werden. In den laufbahnbestimmenden Referatenorganen taucht er zumeist gar nicht auf. Zwischen 1980 und 1996 sinkt der Anteil der deutschen Sprache an der naturwissenschaftlichen Weltpublikation von 3,5 auf 1,2 Prozent, der des Russischen von 10,8 auf 2,1, steigt der des englischen von 74,6 auf 90,7 Prozent. Vergleichbares gilt, wenn auch in verschiedenem Maß, von der Medizin, den Kognitionswissenschaften und der Wirtschaft, den technischen Disziplinen und der technischen Industrie, aber auch zunehmend von kultur- und geisteswissenschaftlichen Fä- 
chern. Indologen, Archäologen, auch Sprachwissenschaftler des Faches Deutsch schreiben heute teilweise Englisch (vgl. Ammon 2000).

Was bedeutet diese Entwicklung? Wie sieht sie sich an, wenn wir auf unsere Sprachgeschichte zurückblicken? Wird unsere Sprache, wenn sie auf den das öffentliche Gebiet bestimmenden Feldern nicht mehr ausgebaut und erweitert wird, wenn sie mit der Weltentwicklung nicht Schritt hält, zu einer Art Dialekt? Dialekte sind ja deshalb so intim, weil sie auf den öffentlichen Feldern nicht mehr weiter ausgebaut wurden.

Wie lässt sich auf die Herausforderung antworten, was könnte sich entwickeln? Ich will versuchen, an das von manchen als bedrohlich angesehene Massiv etwas näher heranzugehen, seine Täler und Höhen und Klüfte genauer ins Auge zu fassen, das Panorama auseinanderzufalten. Die Sache hat viele Seiten, ich versuche das in zehn Punkten zu zeigen. Bevor ich darauf in zehn Thesen eingehe, möchte ich zwei Vorfragen stellen, auf zwei Vorurteile eingehen, von denen ich glaube, dass sie die Sicht behindern.

Ist der Mensch eigentlich von Natur ein einsprachiges Wesen, ein homo monolinguis, der seine Muttersprache hat, durch sie seine Identität gewinnt und was es sonst an Sprachen gibt, ist Fremdsprache? Und ist die angestammte Sprache etwas Einheitliches, ein homogener Werk- und Erbstoff? Der Nationalstaat, insbesondere unser verspäteter Nationalstaat, hat diese Vorstellung hervorgebracht und betont. Die Sprache erschien dem frühen 19. Jahrhundert als das erste, älteste einheitsstiftende Band, und man vermutete: je älter umso reiner. Das ist eine Theorie, die so nicht haltbar ist.

Unser Sprachraum war zu keiner Zeit einsprachig, unsere Sprache wohl niemals eine homogene Einheit. Dass der Mensch von Natur ein ,homo monolinguis‘ ist, ein einsprachiges Wesen, ist ein Mythos des Nationalstaats, und dass es eine ursprünglich reine, unvermischte Sprache gab, ist in historischer Zeit nicht nachweisbar. (Die Inder sind so sprachbegabt, habe ich als Kind oft gehört, ihnen fliegen auf dem Markt mehrere Sprachen zu. Vielleicht sind sie gar nicht so speziell begabt, sondern haben nur nicht die europäische Theorie, die besagt, dass es eine Muttersprache und Fremdsprachen gäbe?)

Sprachen sind geschichtliche Zusammensetzungen - allerdings wohl in verschiedenem Grad zusammengesetzt.

Die andere Frage ist nicht weniger grundsätzlich: was ist eigentlich getroffen, wenn in größerem Maße Worte, Ausdrucksweisen in eine Sprache einwandern, entliehen, übernommen werden: die Sprache oder der Sprachgebrauch? Wir sagen meist ,die Sprache‘, wir haben keine zwei Worte, die das System Sprache und den eingeführten, üblichen, gewohnten oder auch eingerissenen Sprachgebrauch trennen. Die beiden sind auch nicht ganz zu trennen, aber sie sind keineswegs dasselbe. Unsere Sprache als System ist von Entlehnungen nicht 
betroffen; sie kann Vokabeln wie ,pullover‘ und ,strike' so assimilieren, sich so einverleiben, dass man sie als entlehnte Wörter nicht mehr erkennt; wir können sie beugen, die Mehrzahl bilden, zusammensetzen; das gilt auch für das, was zur Zeit neu entlehnt wird. Das System ist nicht erschüttert - die Sprache ist das beweglichste, flexibelste, aufnahmefähigste Sozialgebilde, das der Mensch hat, und darum zugleich das konservativste, widerstandsfähigste. Darum konnte ein Staat wie Israel eine fast 3000 Jahre alte Sprache - in enorm erweiterter Fassung - zur Staatssprache machen. Die Sprache - als System - ist von unerschöpflicher Regenerationskraft. Sie arbeitet mit einem einfachen Kunstgriff sie macht, wie Wilhelm von Humboldt (1979: 477) sagt, von endlichen Mitteln einen unendlichen Gebrauch. Sie arbeitet auf der einen Seite mit flexiblen Einzelzeichen, Symbolen, den Wörtern, und auf der anderen mit flexiblen Verknüpfungsregeln. Mit Lexikon und Grammatik. Schon vierjährige Kinder verstehen den komplexen Satzbau der Grimmschen Märchen. Man braucht sie mit Sätzen wie „tut tut tut - ein Auto“ nicht zu öden. Jetzt zu den Thesen:

\section{Zweisprachigkeit ist in der Geschichte der Menschheit ein Normalfall.}

Wenn man auf die Weltkulturen sieht, ist Zweisprachigkeit keine Ausnahme. Sie galt eben auch für die europäische Kultur. Das Gebiet, auf dem die menschliche Zunge sich deutschsprachig artikulierte, ,theodisce', wie es in einem latinisierenden Gelehrtenwort des 8. Jahrhunderts hieß - das Wort theodiscus, aus dem dann diutisc, diutsch oder tiusch, deutsch wird, bedeutete ,volkssprachig،, ,landessprachlich', ,laiensprachlich“ -, das Gebiet also, wo die menschliche Zunge sich auf unsere laiensprachliche Weise altalemannisch, altfränkisch, altniederdeutsch, altbairisch artikulierte, war vom 8. bis zum 18. Jahrhundert ein Gegenüber von Deutsch und Latein, genauer gesagt von überwiegend mündlich gebrauchten deutschen Dialekten und einer lateinischen Schriftkultur. Die Sprache der Kirche und Klöster, der späteren Universität, der Poesie, des Rechts und der Diplomatie waren lange und dann abnehmend lateinisch. Ein solches Gegenüber von Laiensprache und Mandarinensprache kennen wir aus Arabien und Indien, Indonesien, Tibet oder China.

An einer berühmten Stelle spricht Martin Luther darüber, wie er dazu gekommen sei, sein „gemeines“, d.h. allgemein verständliches Deutsch in seiner Bibelübersetzung und in seinen Flugschriften zu schreiben. In seinen überlieferten Tischreden vom Jahr 1532 liest man:

Ich habe keine bestimmte, spezielle Sprache als mein deutsch, sondern eine gemeinsame, damit mich die aus dem oberen und aus dem niederen Deutschland verstehen können. Ich rede nach der Sächsischen Kanzlei, welche alle Fürsten und Könige Deutschlands nach- 
ahmen. Alle Reichsstätte, Fürstenhöfe schreiben nach der Sächsischen Kanzlei unserer Kurfürsten. Darum ist dies die gemeinschaftlichste Sprache Deutschlands. Kaiser Maximilian und Kurfürst Friedrich haben das Reich so auf eine bestimmte Sprache eingegrenzt: sie haben also alle Sprachen in eine gezogen.

Dieser Text ist allerdings eine Übersetzung, im Originalton klingt er so:

Nullam certam linguam Germanice habeo, sed communem ut me intellegire possint ex superiori et inferiori Germaniae. Ich rede nach der Sechsischen cantzley, quam imitantur omnes duces et reges Germaniae; alle reichstette, fürsten höfe schreiben nach der Sechsischen cantzelein unser churfürsten. Ideo est communissima lingua Germaniae. Maximilianus imperator et elector Fridericus imperium ita ad certam linguam definierunt, haben also alle sprachen in eine getzogen. (Zit. nach von Polenz 2000: 166; Kursivdruck steht hier für Frakturschrift)

Luther war zweisprachig, einmal rein deutsch, einmal rein lateinisch; häufig gemischt.

Von 800 bis 1800 - rund, allzu rund gerechnet 1000 Jahre, war unser Sprachgebiet zweisprachig. „Soviel Europa war nie“, schrieb Kurt Flasch (2005: 44) in einem Aufsatz über Latein und Volgare.

\section{Im 17., 18., 19. Jahrhundert waren wir dreisprachig.}

Das Französische kam hinzu, es hatte im 17. Jahrhundert, zur Zeit Ludwig des XIV. und der Gründung der Académie Française eine enorme Ausstrahlung, man baute und gärtnerte wie in Frankreich und kleidete sich, speiste und parlierte, man liebte Französisch, deutsche Texte waren zeitweise so weitgehend französisch durchsetzt, dass unsere heutige Anglisierung sich dagegen harmlos ausnimmt. Der junge Philosoph Leibniz schrieb 1682/83 eine Ermahnung an die Deutschen, ihren Verstand und ihre Sprache besser zu üben, samt beigefügtem Vorschlag einer deutschgesinnten Gesellschaft.

Ich muß bekennen, es sei leider dahin gekommen, daß man vielleicht, so lange Deutschland steht, nie darin undeutscher und ungereimter geredet hat. Ich rufe zu Zeugen an, was uns die halbjährigen Messen hervorbringen; darin ist oft alles auf eine so erbärmliche Weise durcheinandergeworfen, daß manche sogar nicht einmal zu erwägen scheinen, was sie schreiben. Ja, es scheint, manche dieser Leute haben ihr Deutsch vergessen und Französisch nicht gelernt. Wollte Gott, es wäre jedesmal unter zehn solcher fliegenden Papiere eines, so ein Fremder ohne Lachen, ein Patriot ohne Zorn lesen könne! (Leibniz 1983: 66f.)

Er klagt auf deutsch, aber dies war eine Schrift, die bis 1846 in der Schublade liegen blieb. Leibniz schrieb Lateinisch und Französisch. - Der Dichter Sigmund 
von Birken verfasste ein Spottgedicht auf das Kauderwelsch seiner Zeit (zit. nach Otto 1972: 1):

Ich bin nun dechargirt von dem maladen Leben.

Mir hat der Maur facon genug disgousto geben.

Wo Einfalt avancirt, und Unschuld mit raison,

Die retrogarde hat / da ist die Sache bon.

3 In Anlehnung an das Lateinische und Französische und im Gegenzug dazu hat sich das Deutsche als Kultursprache herausgebildet, unabhängig vom modernen Staat, ohne zentrales Institut, ohne Akademie und Sprachaufsicht. Wir waren auch sprachlich eine Kulturnation, lange bevor es - seit Bismarcks Einigungskriegen - einen deutschen Staat gab.

Wie das geschah, durch wen und was, das wäre eine lange Geschichte, die spannend zu verfolgen ist: in der Zeit der Fürstenkanzleien, der Bauernkriege und ihrer Flugschriften, der lutherischen Reformation und dann, in einem weiteren entscheidenden Schritt, das ganze 18. Jahrhundert hindurch wurde diese deutsche Einheitssprache erreicht (Gottsched war auf diesem Gebiet wirksamer als Goethe).

An dessen Ende haben wir eine auf allen Feldern ausgebaute, differenziert ausgearbeitete und allgemein durchsichtige und verständliche Standardsprache, nicht nur auf dem Gebiet der Dichtung, sondern auch auf dem der Sachgebiete, der Sachprosa. - Allerdings keine „reine“ Sprache.

\section{Der jahrhundertelange Sprachenkontakt mit dem Lateinischen und Französischen hat das Deutsche erweitert und bereichert.}

Es ist überraschend, wenn man erfährt, wie viele als deutsch empfundene Wörter dem Lateinischen entstammen oder ihm nachgebildet sind - Wein und Meister, Zelle und Fenster, oder Einfluss und Einbildung, Anfang und Gegenstand sind Leihgut. Das Wort Objekt wurde zuerst wörtlich wiedergegeben als Gegenwurf.

Als Joachim Heinrich Campe um 1800 sein Wörterbuch der unserer Sprache aufgedrungenen fremden Ausdrücke vorlegte (Campe 1801; 1813), erreichte er, wenn sein deutsches Wort sich durchsetzte, zumeist dass es neben das aus Frankreich entlehnte Wort trat: es gab nebeneinander Journale und Tageszeitungen, eine Struktur und eine Bauart.

Die zur Zeit genaueste und umsichtigste Deutsche Sprachgeschichte sind die drei Bände von Peter von Polenz (1991/1994/1999; 2000). Er sieht im Sprachenkontakt eine „unschätzbare lexikalische und stilistische Bereicherung deutscher 
Sprachkultur“, den zweisprachigen Autoren sei „gerade durch das Französische die Einbeziehung Deutschlands in den modernen westeuropäischen Kulturzusammenhang zu verdanken“ (von Polenz 1994: 49f.).

\section{Unsere Sprache wird sich im angebrochenen Jahrhundert unvermeid- lich internationalisieren - die Offenheit gegenüber dem Anglo-amerika- nischen kann sie auf ihre Zukunft vorbereiten.}

Lehnwörter sind Sprachbrücken. Die Wörter Minorität, Opposition z. B. verbinden uns mit zahlreichen Sprachen.

Wenn wir in den Bereichen, in denen ein internationaler Verkehr selbstverständlich geworden ist, aus dessen Vokabular einiges übernehmen, erleichtern wir den Anschluss: in den Naturwissenschaften, in Technik und Wirtschaft, in dem Warenaustausch, in der elektronischen Kommunikation. Noch einmal ein geschichtliches Beispiel: Das Englische ist keine germanische Sprache; es hat sich dem Lateinischen und Französischen früh geöffnet. Schon die Sprache Chaucers, jenes Dichters des Spätmittelalters, hat zu 38 \% lateinisch-romanische Ausdrücke. In heutigen englischen Texten ist der Anteil oftmals noch höher. Englisch ist eine germanisch-romanische Mischsprache; gerade das, und die einfache Grammatik, macht es als internationales Vehikel geeignet.

\section{Die meisten Entlehnungen sind Eintagsfliegen, etliche eine Bereiche- rung.}

Das Vokabular der Werbung, der Musikszene, zahlreiche Warenbezeichnungen werden vermutlich nur ein kurzes Leben haben; sie verschwinden wieder.

Die Entlehnung aus dem Englischen ist verkaufsträchtig; sie bringt ein Flair von Neuheit, Aktualität, Modernität mit sich. Das könnte eine vorübergehende Mode sein; heute ist es nicht mehr chique, Paraplu und Mademoiselle und contenance zu sagen.

Für „Leihgut“, das langfristig assimiliert wird - (unsere Grammatik erleichtert die Einbürgerung von Lehnwörtern) - gibt es meistens einen Grund; das entlehnte Wort füllt eine Lücke in unserem Wortschatz. Wir müssten eine Umschreibung an seine Stelle setzen: black-box, know-how, Walkman, Insider, (der Binnenseiter wäre möglich, scheint aber nicht zu gehen).

Inter-City - kein übles Wort. Cool ist kaum zu übersetzen oder Okay. Unter dem Appell „Clean ist wieder in“ in der Bonner U-Bahn hat ein verärgerter Fahrgast geschrieben: „Sauber ist wieder gefragt.“ Ist clean - sauber, in - gefragt? Ob dies Wort bleibt - schwer zu sagen. Andererseits, ein großes ANDERERSEITS: 


\section{Wenn die Kernbereiche unserer Gesellschaft englisch und nur noch englisch formuliert werden, wenn das Deutsche in diesen Bezirken nicht ausgebaut wird, verarmt unsere Sprache, und unsere allgemeine Begriffswelt.}

Wenn Technik z.B. und Wirtschaft, Naturwissenschaft und Gesellschaftstheorie und Philosophie sich fast nur noch englisch ausdrücken, wenn ihre zentralen Begriffe nicht mehr allgemein durchsichtig sind, haben wir eine Zweiklassengesellschaft, eine Sprachbarriere innerhalb der Gesellschaft. Und die Landessprache bleibt zurück.

In der Geschichte der Neuzeit hat unsere Gesellschaft, wie angedeutet den umgekehrten Weg eingeschlagen: „Die Wissenschaft ist dem Licht gleich“, schrieb Leibniz (1916a: 86), „und es ist ein allgemeines Interesse, daß es auf alle einzelnen ausgegossen sei.“ Die deutsche Sprachgeschichte der Neuzeit ist nicht nur ein Vorgang der sprachlichen Standardisierung, sondern auch der eines Sprachausbaus in alle Richtungen: Eine Demokratisierung der Sprache. Sie war Joachim Heinrich Campes ursprünglicher Impuls (vgl. Schiewe 1988). - Dieser Prozess ist rückläufig. - Inzwischen werden die Zukunftsfragen unserer Gesellschaft von Naturwissenschaft und Technik, Wirtschaft, Gesellschaftsanalyse und Theorie entschieden. Politik und Öffentlichkeit brauchen für ihre Beratung eine allgemein durchsichtige Sprache.

Auf diesen Gebieten hatten wir im 18., 19., 20. Jahrhundert eine großartige deutschsprachige Literatur: von den Humboldts über Helmholtz, Hertz, Haeckel, Tönnies, Freud bis zu Einstein, Heisenberg, Max Weber. Einstein (1953) schrieb z.B.: „Nach unserer bisherigen Erfahrung sind wir nämlich zum Vertrauen berechtigt, dass die Natur die Realisierung des mathematisch denkbar Einfachsten ist.“ Ein klassisch schönes Deutsch. - Mittlerweile ist eine Kluft entstanden.

Unser Sprach- und Begriffshorizont sollte nicht hinter den Entwicklungen hinterherhinken. Wie wäre das zu bewerkstelligen?

\section{Nicht durch staatliche, zentral gesteuerte Aktivität. Die staatsunabhän- gige Herausbildung unserer reichen durchsichtigen Standardsprache ist eine zu wertvolle Erfahrung.}

Und was der Staat an- und auszurichten imstande ist - bei uns! anderswo mag das anders sein - hat er gerade auf dem Gebiet der Rechtschreibung unter Beweis gestellt. Er hat eine fehlerhafte Neuregelung der Rechtschreibung zu einem Zeitpunkt durchgesetzt, als kaum zu widerlegende Kritik auf dem Tisch lag. Ich würde nicht nach dem Staat rufen, nicht die Staatsbürokratie an die Sprache heranlassen. Sie ist wie der Eber im Weinberg. 


\section{Die wichtigsten Werkzeuge sind nach meinem Eindruck vorhanden, aber nicht intensiv genug genutzt:}

- Das erste ist der Sprachunterricht im Deutschen und Englischen, und, wie unser früherer Freiburger Kollege Wulf Oesterreicher (2005) vorschlägt, einer weiteren unmittelbaren Nachbarsprache. Dass mit Schulbeginn eine zweite Sprache von Anfang an spielerisch aufgebaut werden kann, ist z.B. eine Möglichkeit. Ich will mich hier auf das enorme Gebiet der Praxis nicht einlassen, nur die Vermutung aussprechen: je intensiver, gründlicher, differenzierter die andere Sprache erworben wird, gerade das Englische - es hat einen enorm reichen, nuancierten, Abstraktes konkretisierenden Wortschatz -, umso mehr profitiert die eigene Sprache, wird sie aufgebaut und erweitert.

- Das zweite Mittel ist die Sachliteratur, das sachorientierte Hörbuch, der sachorientierende Film. Hier steht ein enormes Potential zur Verfügung. Die Populärwissenschaft ist ein Gebiet, das Wissenschaft übersetzt, in sie einführt. Das kann sehr unterhaltend und belehrend sein.

Das größte Defizit ist hier seit einiger Zeit bei den staatlich begünstigten öffentlich-rechtlichen Rundfunk- und Fernsehanstalten zu verzeichnen. Sie haben die Aufgabe, nicht nur zu unterhalten, sondern auch aufzuklären, zu belehren und zu bilden. Dafür werden sie unterstützt. In ihnen ist das Wort längst auf dem Rückzug. Sie unterfordern durch Tralalakultur und Unterhaltungszickzack hemmungslos das Publikum. Die Unterbindung wortzentrierter Sendungen ist kein Marktschicksal, sondern ein Staatsversagen, weil das öffentlich-rechtliche Medium auf diese Weise den Übergang in eine bildungsschwache Stimmungsdemokratie begünstigt, $d$. h. seine übernommene Aufgabe vernachlässigt.

- Eine Möglichkeit, die am erfolgreichsten durch ein Übersetzerkolleg zu fördern wäre, sehe ich in der Selbstübersetzung hervorragender Wissenschaftler, deren Fach ins Englische übergegangen ist. Sie könnten am besten dafür sorgen, dass die Begriffssprache ihres Faches nicht nur auf Englisch, sondern auch, wo sie von allgemeinem Belang ist, in unserer Sprache öffentlich vorhanden ist. Sie könnten am besten für den Sprachausbau sorgen, der mit der Weltentwicklung Schritt hält.

- Sie verstehen, wie es gemeint ist, wenn ich sage: die gegenwärtige Veränderung unseres Sprachgebrauchs durch die Übernahme englischen, allgemeiner gesagt internationalen Vokabulars ist keine Gefahr, sondern eher eine Chance, eine Notwendigkeit.

- Die letzte, nicht zu unterschätzende Instanz ist die Schöne Literatur. Sie kann sich, z.B. in der Poesie, ganz freihalten vom Fremdwort, kann aber mit dieser ,silbernen Rippe des Sprachleibs', wie Walter Benjamin (1928: 62) es genannt hat, auch die faszinierendste Wirkung erzielen. Sie ist der Ort der größt- 
möglichen Synthese, der Reichweite in jeder Richtung. Sie erhält altes Wortgut, das sonst vergessen würde. Sie erweitert die Gegenwartssprache durch Bildungen neuer Wörter, die genau und lebensfähig sind. Sie bereichert den Wortbestand durch gelungene Übersetzungen aus anderen Sprachen. Sie ist die vielleicht vorsichtigste, behutsamste Instanz, wenn es darum geht, entlehnte Wörter heimisch zu machen, sie in durchsichtigem Zusammenhang zu assimilieren, ihnen Heimatrecht zu geben, den Schrott auszuscheiden, zu sichten, sprachliche Orientierung zu geben. Dem vergangenen Jahrhundert fehlt es dafür nicht an Beispielen - auch nicht an Wissenschaftlern, die zur Literatur gehören. Sprache steht nicht still. Literatur gehört zu den wichtigsten Instanzen, die sie erhält und erweitert: sie entwickelt. Ihre stärksten Vertreter zeichnen sich aus durch ein fein reagierendes Sprachgewissen und eine Saugkraft, welche sprachliche Vielfalt anzieht.

\title{
10 Zum Schluss möchte ich noch einmal an die Weimarer Exzellenz er- innern und an ihre Entschiedenheit:
}

„Ich verfluche allen negativen Purismus, daß man ein Wort nicht brauchen soll, in welchem eine andre Sprache Vieles oder Zarteres gefaßt hat.“

„Die Gewalt einer Sprache ist nicht, daß sie das Fremde abweist, sondern daß sie es verschlingt.“ (Goethe 1907: 207)

Oder, was vielleicht noch entscheidender ist: an die produktive Unruhe, mit der Goethe dem treffenden Wort auf der Spur ist:

\author{
Worte sind der Seele Bild - \\ Nicht ein Bild! sie sind ein Schatten! \\ Sagen herbe, deuten mild \\ Was wir haben, was wir hatten. - \\ Was wir hatten wo ist's hin? \\ Und was ist's denn was wir haben? - \\ Nun, wir sprechen! Rasch im Fliehn \\ Haschen wir des Lebens Gaben.
}

